Article

\title{
On Approximation by Linear Combinations of Modified Summation Operators of Integral Type in Orlicz Spaces
}

\author{
Ling-Xiong Han ${ }^{1}\left(\mathbb{D}\right.$ and Feng $\mathrm{Qi}^{2,3, *(\mathbb{C})}$ \\ 1 College of Mathematics, Inner Mongolia University for Nationalities, Tongliao 028043, Inner Mongolia, \\ China; hlx2980@163.com \\ 2 Institute of Mathematics, Henan Polytechnic University, Jiaozuo 454010, Henan, China \\ 3 School of Mathematical Sciences, Tianjin Polytechnic University, Tianjin 300387, China \\ * Correspondence: qifeng618@gmail.com
}

Received: 11 October 2018; Accepted: 6 December 2018; Published: 21 December 2018

Abstract: In this paper, the authors introduce the Orlicz spaces corresponding to the Young function and, by virtue of the equivalent theorem between the modified $K$-functional and modulus of smoothness, establish the direct, inverse, and equivalent theorems for linear combinations of modified summation operators of integral type in the Orlicz spaces.

Keywords: approximation; linear combination; direct theorem; inverse theorem; equivalent theorem; Orlicz space; modified summation operators of integral type; $K$-functional; modulus

MSC: 41A17; 41A27; 41A35

\section{Introduction and Main Results}

Throughout this paper, we use $C$ to denote an absolute constant independent of anything, which may be not necessarily the same in different cases.

There are many types of integral operators (see, for example, [1-7] and closely related references therein). In the paper [8], Ueki provided a characterization for the boundedness and compactness of the Li-Stević type integral operators

$$
T_{\varphi}^{g} f(z)=\int_{0}^{z} f(\varphi(\xi)) g(\xi) \mathrm{d} \xi
$$

from the weighted Bergman space $L_{a}^{p}\left(d A_{\alpha}\right)$ to the $\beta$-Zygmund space $Z_{\beta}$. Later, Li and Ma [9] investigated the boundedness and compactness of products of composition operators and integral type operators

$$
\left(C_{\varphi} I_{g} f\right)(z)=\int_{0}^{\varphi(z)} f^{\prime}(\xi) g(\xi) \mathrm{d} \xi
$$

from Zygmund-type spaces to $Q_{k}$ spaces. Recently, the equivalent characterizations for the boundedness and compactness of several integral type operators

$$
V_{\Phi}^{h} f(z)=\int_{0}^{z} f^{\prime}(\Phi(t)) h^{\prime}(t) \mathrm{d} t
$$


from $F(p, q, s)$ space to $\alpha$-Bloch-Orlicz and $\beta$-Zygmund-Orlicz spaces were developed in [10]. Gupta and Yadav [11] estimated the approximation by complex summation integral type operator

$$
M_{n}(f, z)=(n+1) \sum_{k=1}^{n} p_{n, k}(z) \int_{0}^{1} f(t) p_{n, k-1}(t) \mathrm{d} t+f(0) p_{n, 0}(z)
$$

in compact disks, where $p_{n, k}(z)=\left(\begin{array}{l}n \\ k\end{array}\right) z^{k}(1-z)^{n-k}$. In [8-11], some approximating properties of integral type operators in complex spaces were established. Vural, Altin, and Yüksel [12] provided weighted approximation and obtained a rate of convergence of Schurer's generalization of the $q$-Hybrid summation operators of integral type

$$
\begin{aligned}
M_{n, p, q}^{(\alpha, \beta)}(f, x)= & {[n+p-1]_{q} \sum_{k=1}^{\infty} S_{n, p, k}^{q}(x) \int_{0}^{\infty} P_{n, p, k-1}^{q}(t) f\left(\frac{[n+p]_{q} t+\alpha}{[n+p]_{q}+\beta}\right) \mathrm{d}_{q}(t) } \\
& +e^{-[n+p]_{q} x} f\left(\frac{\alpha}{[n+p]_{q}+\beta}\right)
\end{aligned}
$$

where

$$
S_{n, p, k}^{q}(x)=\left([n+p]_{q} x\right)^{k} \frac{e^{-[n+p]_{q} x}}{[k]_{q} !} \quad \text { and } \quad P_{n, p, k}^{q}=\left(\begin{array}{c}
n+p+k-1 \\
k
\end{array}\right)_{q} q^{k(k+1)} \frac{x^{k}}{(1+x)_{q}^{n+p+k}} .
$$

In [13], Govil and Gupta considered the simultaneous approximation for the Stancu-type generalization of certain summation operators of integral type

$$
G_{n}^{\alpha, \beta}(f, x)=\sum_{k=1}^{\infty} p_{n, k}(x) \int_{0}^{\infty} b_{n, k-1}(t) f\left(\frac{n t+\alpha}{n+\beta}\right) \mathrm{d} t+(1+x)^{-n} f\left(\frac{\alpha}{n+\beta}\right)
$$

by hypergeometric series. Srivastava and Gupta [14] introduced and investigated a new sequence of linear positive operators

$$
G_{n, c}(f, x)=n \sum_{k=1}^{\infty} \int_{0}^{\infty} p_{n, k}(x ; c) p_{n+c, k-1}(t ; c) f(t) \mathrm{d} t+\int_{0}^{\infty} p_{n, 0}(x ; c) p_{n, 0}(t ; c) \delta(t) f(t) \mathrm{d} t,
$$

which included some well-known operators as its special cases and obtained an estimate on the rate of convergence by means of the decomposition technique for functions of bounded variation. In [15], Gupta, Mohapatra, and Finta studied the mixed summation operators of integral type

$$
S_{n}(f, x)=\sum_{v=1}^{\infty} s_{n, v}(x) \int_{0}^{\infty} b_{n, v-1}(t) f(t) \mathrm{d} t+e^{-n x} f(0)
$$

and obtained the rate of point-wise convergence, where

$$
s_{n, v}(x)=e^{-n x} \frac{(n x)^{v}}{v !} \quad \text { and } \quad b_{n, v}(t)=\frac{1}{B(n, v+1)} t^{v}(1+t)^{-n-v-1} .
$$

In [12-15], some approximating properties of integral type operators were discussed in $C[0, \infty)$, which is a special case of the Orlicz space. For $f \in L_{\Phi}^{*}[0, \infty)$, the modified summation operators of integral type $B_{n}(f, x)$ are defined in [16] as

$$
B_{n}(f, x)=\frac{1}{n+1} \sum_{k=1}^{\infty} b_{n, k}(x) \int_{0}^{\infty} b_{n, k}(t) f(t) \mathrm{d} t, \quad x \in[0, \infty),
$$


where $b_{n, k}(x)=\frac{(n+k) !}{(k-1) ! n !} \frac{x^{k-1}}{(1+x)^{n+k+1}}$ for $k, n \geq 1$. Recently, Han and $\mathrm{Wu}$ [16] obtained the following direct, inverse, and equivalent theorems of modified summation operators of integral type in Orlicz spaces.

Theorem 1 (Direct theorem [16]). Let $f \in L_{\Phi}^{*}[0, \infty), \varphi^{2}(x)=x(1+x)$, and $\Psi \in \Delta_{2}$. Then

$$
\left\|B_{n}(f)-f\right\|_{\Phi} \leq C\left[\omega_{2, \varphi}\left(f, \frac{1}{\sqrt{n}}\right)_{\Phi}+\omega_{1}\left(f, \frac{1}{n}\right)_{\Phi}+\frac{\|f\|_{\Phi}}{n}\right] .
$$

Theorem 2 (Inverse theorem [16]). Let $f \in L_{\Phi}^{*}[0, \infty), 0 \leq \alpha<2$, and $\left\|B_{n}(f)-f\right\|_{\Phi}=O\left(n^{-\alpha / 2}\right)$. Then

$$
\omega_{2, \varphi}(f, t)_{\Phi}=O\left(t^{\alpha}\right) \text { and } \omega_{1}(f, t)_{\Phi}=O\left(t^{\alpha / 2}\right) \text {. }
$$

Theorem 3 (Equivalence theorem [16]). Let $f \in L_{\Phi}^{*}[0, \infty)$ and $0 \leq \alpha<2$. Then

$$
\left\|B_{n}(f)-f\right\|_{\Phi}=O\left(n^{-\alpha / 2}\right) \quad \text { if and only if } \quad \omega_{2, \varphi}(f, t)_{\Phi}=O\left(t^{\alpha}\right) \quad \text { and } \quad \omega_{1}(f, t)_{\Phi}=O\left(t^{\alpha / 2}\right) .
$$

In recent years, since the Orlicz spaces are more general than the classical $L_{p}$ spaces, which is composed of measurable functions $f(x)$ such that $|f(x)|^{p}$ are integrable, there is growing interest in problems of approximation in Orlicz spaces.

For smoothly proceeding, we recall from [17] some definitions and related results.

A continuous convex function $\Phi(t)$ on $[0, \infty)$ is called a Young function if it satisfies

$$
\lim _{t \rightarrow 0^{+}} \frac{\Phi(t)}{t}=0 \text { and } \lim _{t \rightarrow \infty} \frac{\Phi(t)}{t}=\infty .
$$

For a given Young function $\Phi(t)$, its complementary Young function is denoted by $\Psi(t)$.

It is clear that the convexity of $\Phi(t)$ can lead to $\Phi(\alpha t) \leq \alpha \Phi(t)$ for $\alpha \in[0,1]$. In particular, one has $\Phi(\alpha t)<\alpha \Phi(t)$ for $\alpha \in(0,1)$.

A Young function $\Phi(t)$ is said to satisfy the $\Delta_{2}$-condition, denoted by $\Phi \in \Delta_{2}$, if there exist $t_{0}>0$ and $C \geq 1$ such that $\Phi(2 t) \leq C \Phi(t)$ for $t \geq t_{0}$.

Let $\Phi(t)$ be a Young function. We define the Orlicz class $L_{\Phi}[0, \infty)$ as the collection of all Lebesgue measurable functions $u(x)$ on $[0, \infty)$. Since the integral

$$
\rho(u, \Phi)=\int_{0}^{\infty} \Phi(|u(x)|) \mathrm{d} x
$$

is finite, we define the Orlicz space $L_{\Phi}^{*}[0, \infty)$ as the linear hull of $L_{\Phi}[0, \infty)$ under the Luxemburg norm

$$
\|u\|_{(\Phi)}=\inf _{\lambda>0}\left\{\lambda: \rho\left(\frac{u}{\lambda}, \Phi\right) \leq 1\right\}
$$

The Orlicz norm, which is equivalent to the Luxemburg norm on $L_{\Phi}^{*}[0, \infty)$, is given by

$$
\|u\|_{\Phi}=\sup _{\rho(v, \Psi) \leq 1}\left|\int_{0}^{\infty} u(x) v(x) \mathrm{d} x\right|
$$

and satisfies

$$
\|u\|_{(\Phi)} \leq\|u\|_{\Phi} \leq 2\|u\|_{(\Phi)} .
$$

For $f \in L_{\Phi}^{*}[0, \infty)$, the weighted $K$-functional $K_{r, \varphi}\left(f, t^{r}\right)$, the modified weighted $K$-functional $\bar{K}_{r, \varphi}\left(f, t^{r}\right)$, and the weighted modulus of smoothness $\omega_{r, \varphi}(f, t)_{\Phi}$ are given, respectively, by

$$
K_{r, \varphi}\left(f, t^{r}\right)_{\Phi}=\inf _{g}\left\{\|f-g\|_{\Phi}+t^{r}\left\|\varphi^{r} g^{(r)}\right\|_{\Phi}: g^{(r-1)} \in A C_{l o c}\right\}
$$




$$
\bar{K}_{r, \varphi}\left(f, t^{r}\right)_{\Phi}=\inf _{g}\left\{\|f-g\|_{\Phi}+t^{r}\left\|\varphi^{r} g^{(r)}\right\|_{\Phi}+t^{2 r}\left\|g^{(r)}\right\|_{\Phi}: g^{(r-1)} \in A C_{l o c}\right\}
$$

and

$$
\omega_{r, \varphi}(f, t)_{\Phi}=\sup _{0<h \leq t}\left\|\Delta_{h \varphi}^{r} f\right\|_{\Phi}
$$

where $\varphi(x)=\sqrt{x}, \varphi(x)=\sqrt{x(1+x)}$, or $\varphi(x)=x$, and $g^{(r-1)} \in A C_{l o c}$ means that $g$ is $r-1$ times differentiable and $g^{(r-1)}$ is absolutely continuous in every closed finite interval $[c, d] \subseteq[0, \infty)$.

Between the weighted modulus of smoothness and the modified weighted K-functional, there exists the following equivalent theorem.

Theorem 4 ([16]). Let $f \in L_{\Phi}^{*}[0, \infty)$. Then there exist some constants $C$ and $t_{0}$ such that

$$
\frac{\omega_{r, \varphi}(f, t)_{\Phi}}{C} \leq \bar{K}_{r, \varphi}\left(f, t^{r}\right)_{\Phi} \leq C \omega_{r, \varphi}(f, t)_{\Phi,} \quad 0<t \leq t_{0} .
$$

Between the weighted modulus of smoothness and the weighted $K$-functional, there exists the following equivalent theorem.

Theorem 5 ([18]). Let $f \in L_{\Phi}^{*}[0, \infty)$. Then there are some constants $C$ and $t_{0}$ such that

$$
\frac{\omega_{r, \varphi}(f, t)_{\Phi}}{C} \leq K_{r, \varphi}\left(f, t^{r}\right)_{\Phi} \leq C \omega_{r, \varphi}(f, t)_{\Phi,} \quad 0<t \leq t_{0}
$$

Currently, there are few results about linear combinations of modified summation operators of integral type $B_{n}(f, x)$. In this article, we investigate the approximation of linear combinations of modified summation operators of integral type $B_{n}(f, x)$ in Orlicz spaces $L_{\Phi}^{*}[0, \infty)$. The linear combinations of modified summation operators of integral type $B_{n}(f, x)$ are defined as

$$
L_{n, r}(f, x)=\sum_{i=0}^{2 r-1} c_{i}(n) B_{n_{i}}(f, x)
$$

where

$$
\begin{gathered}
n \leq n_{0} \leq n_{1}<\cdots<n_{2 r-1} \leq C_{n}, \quad \sum_{i=0}^{2 r-1} c_{i}(n)=1, \quad \sum_{i=0}^{2 r-1}\left|c_{i}(n)\right|<C, \\
\sum_{i=0}^{2 r-1} c_{i}(n) B_{n_{i}}\left((t-x)^{k}, x\right)=0, \quad k=0,1,2, \ldots, 2 r-1 .
\end{gathered}
$$

Our main results in this paper can be stated as the following three theorems.

Theorem 6 (Direct theorem). Let $f \in L_{\Phi}^{*}[0, \infty), n \in \mathbb{N}, \Psi \in \Delta_{2}$, and $\varphi(x)=\sqrt{x(1+x)}$. Then

$$
\left\|L_{n, r}(f)-f\right\|_{\Phi} \leq C \omega_{2 r, \varphi}\left(f, \frac{1}{\sqrt{n}}\right)_{\Phi} .
$$

Theorem 7 (Inverse theorem). Let $f \in L_{\Phi}^{*}[0, \infty), n \geq 2 r$, and $\varphi^{2}(x)=x(1+x)$. Then

$$
\omega_{2 r, \varphi}\left(f, \frac{1}{n^{r / 2}}\right)_{\Phi} \leq \frac{C}{n^{r}} \sum_{k=1}^{n} k^{r-1}\left\|L_{n, r}(f)-f\right\|_{\Phi}
$$

Theorem 8 (Equivalent theorem). Let $f \in L_{\Phi}^{*}[0, \infty), n \geq 2 r, \varphi^{2}(x)=x(1+x)$, and $\Psi \in \Delta_{2}$. Then

$$
\left\|L_{n, r}(f)-f\right\|_{\Phi}=O\left(\psi\left(\frac{1}{n^{1 / 2}}\right)\right), \quad n \rightarrow \infty \quad \text { if and only if } \omega_{2 r, \varphi}(f, t)_{\Phi}=O(\psi(t)), \quad t \rightarrow 0^{+} .
$$


These main results improve some conclusions in [19] and increase the approximating speed of corresponding operators.

\section{Proof of the Direct Theorem}

In order to prove the direct theorem, we need several lemmas below.

Lemma 1. The modified summation operator of integral type $B_{n}(f, x)$ defined in Equation (1) satisfies

$$
B_{n}(1, x)=1 \quad \text { and } \quad B_{n}\left((t-x)^{2 r}, x\right) \leq C\left[\frac{\delta_{n}^{2}(x)}{n}\right]^{r},
$$

where $\delta_{n}^{2}(x)=\max \left\{\varphi^{2}(x), \frac{1}{n}\right\}, \varphi(x)=\sqrt{x(1+x)}, r \in \mathbb{N}$, and $C$ is a positive constant.

Proof. This follows from simple calculation.

Lemma 2 ([19]). If $u$ locates between $x$ and $t$, then

$$
\frac{(t-u)^{2 r-1}}{\varphi^{2 r}(u)} \leq \frac{|t-x|^{2 r-1}}{\varphi^{2(r-1)}(x)} \frac{1}{x}\left(\frac{1}{1+x}+\frac{1}{1+t}\right) .
$$

Lemma 3. Let $f \in L_{\Phi}^{*}[0, \infty)$. Then

$$
\left\|L_{n, r}(f)\right\|_{\Phi} \leq C\|f\|_{\Phi}
$$

Proof. By Lemma 3.2 in [16], we have

$$
\left\|B_{n}(f)\right\|_{\Phi} \leq 2\|f\|_{\Phi}
$$

Using Equation (5), we obtain

$$
\left\|L_{n, r}(f)\right\|_{\Phi}=\left\|\sum_{i=0}^{2 r-1} c_{i}(n) B_{n_{i}}(f)\right\|_{\Phi} \leq \sum_{i=0}^{2 r-1}\left|c_{i}(n)\right|\left\|B_{n_{i}}(f)\right\|_{\Phi} \leq C\|f\|_{\Phi} .
$$

The proof of Lemma 3 is complete.

Lemma 4 ([18]). For $f \in L_{\Phi}^{*}[0, \infty)$ and $\Psi \in \Delta_{2}$, we have

$$
\|\theta(f)\|_{\Phi} \leq C\|f\|_{\Phi}
$$

where

$$
\theta(f, x)=\sup _{\substack{0 \leq t<\infty \\ t \neq x}} \frac{1}{t-x} \int_{x}^{t} f(u) \mathrm{d} u
$$

is the Hardy-Littlewood function of $f(x)$, and $C$ is a positive constant.

We are now in a position to prove Theorem 6.

Proof of Theorem 6. Let

$$
U=W_{\Phi}^{2 r}\left\{g: g^{(2 r-1)} \in A C_{l o c}, \varphi^{2 r} g^{(2 r)} \in L_{\Phi}^{*}[0, \infty)\right\} .
$$


Taylor's formula with integral remainder of $g \in U$ reads

$$
g(t)=\sum_{i=0}^{2 r-1} \frac{g^{(i)}(x)}{i !}(t-x)^{i}+R_{2 r}(g, t, x)
$$

where

$$
R_{2 r}(g, t, x)=\frac{1}{(2 r-1) !} \int_{x}^{t}(t-u)^{2 r-1} g^{(2 r)}(u) \mathrm{d} u, \quad x \in[0, \infty) .
$$

From Equation (5), it follows that $L_{n, r}(g, x)-g(x)=L_{n, r}\left(R_{2 r}(g, t, x), x\right)$ and

$$
\left\|L_{n, r}(g)-g\right\|_{\Phi}=\left\|L_{n, r}\left(R_{2 r}(g, \cdot, x), x\right)\right\|_{\Phi} .
$$
leads to

Now we estimate $\left|R_{2 r}(g, t, x)\right|$. As $x \in\left[\frac{1}{n}, \infty\right)$, we have $\delta_{n}^{2}(x)=\varphi^{2}(x)$. Applying Lemma 2

$$
\begin{aligned}
\left|R_{2 r}(g, t, x)\right| & \leq \frac{1}{(2 r-1) !}\left|\int_{x}^{t} \frac{(t-u)^{2 r-1}}{\varphi^{2 r}(u)} \delta_{n}^{2 r}(u) g^{(2 r)}(u) \mathrm{d} u\right| \\
& \leq \frac{1}{(2 r-1) !} \frac{|t-x|^{2 r-1}}{\varphi^{2 r-2}(x)} \frac{1}{x}\left(\frac{1}{1+x}+\frac{1}{1+t}\right)\left|\int_{x}^{t} \delta_{n}^{2 r}(u) g^{(2 r)}(u) \mathrm{d} u\right| \\
& \leq \frac{1}{(2 r-1) !} \frac{(t-x)^{2 r}}{\varphi^{2 r-2}(x)}\left[\frac{1}{x(1+x)}+\frac{1}{x(1+t)}\right]\left|\theta\left(\delta_{n}^{2 r} g^{(2 r)}, x\right)\right| \\
& \triangleq I_{1}+I_{2} .
\end{aligned}
$$

From Lemma 1, we conclude that

$$
\begin{gathered}
B_{n}\left(I_{1}, x\right)=\frac{1}{n+1} \sum_{k=1}^{\infty} b_{n, k}(x) \int_{0}^{\infty} b_{n, k}(t) \frac{1}{(2 r-1) !} \frac{(t-x)^{2 r}}{\varphi^{2 r}(x)}\left|\theta\left(\delta_{n}^{2 r} g^{(2 r)}, x\right)\right| \mathrm{d} t \\
=\frac{\left|\theta\left(\delta_{n}^{2 r} g^{(2 r)}, x\right)\right| \varphi^{-2 r}(x)}{(2 r-1) !} B_{n}\left((t-x)^{2 r}, x\right) \leq \frac{C}{n^{r}}\left|\theta\left(\delta_{n}^{2 r} g^{(2 r)}, x\right)\right|
\end{gathered}
$$

and

$$
\begin{aligned}
B_{n}\left(I_{2}, x\right) & =\frac{\left|\theta\left(\delta_{n}^{2 r} g^{(2 r)}, x\right)\right|}{n+1} \sum_{k=1}^{\infty} b_{n, k}(x) \int_{0}^{\infty} b_{n, k}(t) \frac{1}{(2 r-1) !} \frac{(t-x)^{2 r}}{\varphi^{2 r-2}(x)} \frac{1}{x(1+t)} \mathrm{d} t \\
& =\frac{\left|\theta\left(\delta_{n}^{2 r} g^{(2 r)}, x\right)\right| \varphi^{-2 r}(x)}{(2 r-1) !(n+1)} \sum_{k=1}^{\infty}\left(\frac{n+1}{n+k+1}\right)^{2} b_{n+1, k}(x) \int_{0}^{\infty} b_{n+1, k}(t)(t-x)^{2 r} \mathrm{~d} t \\
& \leq \frac{C}{n^{r}}\left|\theta\left(\delta_{n}^{2 r} g^{(2 r)}, x\right)\right| .
\end{aligned}
$$

Hence, by Inequalities (7) and (8) and Lemma 4, it follows that

$$
\left\|B_{n}\left(R_{2 r}(g, \cdot, x), x\right)\right\|_{\Phi\left[\frac{1}{n}, \infty\right)} \leq \frac{C}{n^{r}}\left\|\theta\left(\delta_{n}^{2 r} g^{(2 r)}, x\right)\right\|_{\Phi\left[\frac{1}{n}, \infty\right)} \leq \frac{C}{n^{r}}\left\|\delta_{n}^{2 r} g^{(2 r)}\right\|_{\Phi\left[\frac{1}{n}, \infty\right)} .
$$

For $x \in\left[0, \frac{1}{n}\right)$ and $\delta_{n}^{2}(x)=\frac{1}{n}$, we have

$$
\begin{gathered}
\left|R_{2 r}(g, t, x)\right|=\frac{1}{(2 r-1) !}\left|\int_{x}^{t}(t-u)^{2 r-1} g^{(2 r)}(u) \mathrm{d} u\right| \\
=\frac{1}{(2 r-1) !}\left|\int_{x}^{t} \frac{(t-u)^{2 r-1}}{1 / n^{r}} \delta_{n}^{2 r}(u) g^{(2 r)}(u) \mathrm{d} u\right| \leq \frac{1}{(2 r-1) !}(x-t)^{2 r} n^{r}\left|\theta\left(\delta_{n}^{2 r} g^{(2 r)}, x\right)\right| .
\end{gathered}
$$


Using Lemmas 1 and 4 arrives at

$$
B_{n}\left(\left|R_{2 r}(g, t, x)\right|, x\right) \leq \frac{n^{r}}{(2 r-1) !} \frac{C}{n^{r}} \delta_{n}^{2 r}(x)\left|\theta\left(\delta_{n}^{2 r} g^{(2 r)}, x\right)\right| \leq \frac{C}{n^{r}}\left|\theta\left(\delta_{n}^{2 r} g^{(2 r)}, x\right)\right|
$$

and

$$
\left\|B_{n}\left(R_{2 r}(g, \cdot, x), x\right)\right\|_{\Phi\left[0, \frac{1}{n}\right)} \leq \frac{C}{n^{r}}\left\|\theta\left(\delta_{n}^{2 r} g^{(2 r)}\right)\right\|_{\Phi\left[0, \frac{1}{n}\right)} \leq \frac{C}{n^{r}}\left\|\delta_{n}^{2 r} g^{(2 r)}\right\|_{\Phi\left[0, \frac{1}{n}\right)} .
$$

Combining this with Equation (9) leads to

$$
\left\|B_{n}\left(R_{2 r}(g, \cdot, x), x\right)\right\|_{\Phi[0, \infty)} \leq \frac{C}{n^{r}}\left\|\delta_{n}^{2 r} g^{(2 r)}\right\|_{\Phi[0, \infty)}
$$

and, consequently,

$$
\begin{aligned}
\left\|L_{n, r}\left(R_{2 r}(g, \cdot x), x\right)\right\|_{\Phi} \leq \sum_{i=0}^{2 r-1}\left|c_{i}\right|\left\|B_{n_{i}}\left(R_{2 r}(g, \cdot x), x\right)\right\|_{\Phi} & \\
& \leq \sum_{i=0}^{2 r-1}\left|c_{i}\right| \frac{C}{n_{i}^{r}}\left\|\delta_{n_{i}}^{2 r} g^{(2 r)}\right\|_{\Phi} \leq \sum_{i=0}^{2 r-1}\left|c_{i}\right| \frac{C}{n^{r}}\left\|\delta_{n}^{2 r} g^{(2 r)}\right\|_{\Phi} \leq \frac{C}{n^{r}}\left\|\delta_{n}^{2 r} g^{(2 r)}\right\|_{\Phi} .
\end{aligned}
$$

Then, applying the above inequality, Inequalities (3) and (6), and Lemma 3, we obtain

$$
\begin{aligned}
\left\|L_{n, r}(f)-f\right\|_{\Phi} \leq\left\|L_{n, r}(f-g)-(f-g)\right\|_{\Phi} & +\left\|L_{n, r}(g)-g\right\|_{\Phi} \\
& \leq C\|f-g\|_{\Phi}+\frac{C}{n^{r}}\left\|\delta_{n}^{2 r} g^{(2 r)}\right\|_{\Phi} \leq C \omega_{2 r, \varphi}\left(f, \frac{1}{\sqrt{n}}\right)_{\Phi} .
\end{aligned}
$$

The proof of the direct theorem is complete.

\section{Proofs of the Inverse and Equivalent Theorems}

For proving Theorems 7 and 8, we need the following lemmas.

Lemma 5. If $f \in L_{\Phi}^{*}[0, \infty)$ and $n \geq 2 r$, then

$$
\left\|\varphi^{2 r} L_{n, r}^{(2 r)}(f)\right\|_{\Phi} \leq C n^{r}\|f\|_{\Phi}
$$

Proof. Since

$$
\begin{aligned}
B_{n}^{(2 r)}(f, x) & =\frac{1}{n+1} \sum_{k=1}^{\infty} b_{n, k}^{(2 r)}(x) \int_{0}^{\infty} b_{n, k}(t) f(t) \mathrm{d} t \\
= & \frac{1}{n+1} \sum_{k=1}^{\infty} \frac{(n+k) !}{(k-1) ! n !} \sum_{i=0}^{2 r}\left(\begin{array}{c}
2 r \\
i
\end{array}\right)(-1)^{i} D^{2 r-i}\left(x^{k-1}\right) D^{i}\left(\frac{1}{(1+x)^{n+k}}\right) \int_{0}^{\infty} b_{n, k}(t) f(t) \mathrm{d} t \\
= & \prod_{j=2}^{2 r}(n+j) \sum_{k=1}^{\infty} b_{n+2 r, k}(x) \int_{0}^{\infty} \sum_{i=0}^{2 r}\left(\begin{array}{c}
2 r \\
i
\end{array}\right)(-1)^{i} b_{n, k+2 r-i}(t) f(t) \mathrm{d} t \\
\leq & \prod_{j=2}^{2 r}(n+j) \sum_{k=1}^{\infty} b_{n+2 r, k}(x) \int_{0}^{\infty} \sum_{i=0}^{2 r}\left(\begin{array}{c}
2 r \\
i
\end{array}\right) b_{n, k+2 r-i}(t) f(t) \mathrm{d} t,
\end{aligned}
$$

we have

$$
\varphi^{2 r}(x) B_{n}^{(2 r)}(f, x) \leq \prod_{j=2}^{2 r}(n+j) \sum_{k=1}^{\infty} b_{n+2 r, k} \varphi^{2 r}(x) \int_{0}^{\infty} \sum_{i=0}^{2 r}\left(\begin{array}{c}
2 r \\
i
\end{array}\right) b_{n, k+2 r-i}(t) f(t) \mathrm{d} t
$$




$$
\begin{aligned}
& \leq \prod_{j=2}^{2 r}(n+j) \sum_{k=1}^{\infty} b_{n, k+r}(x) \frac{(k+r-1) ! n !}{(n+r+k) !} \frac{(n+2 r+k) !}{(k-1) !(n+2 r) !} \int_{0}^{\infty} \sum_{i=0}^{2 r}\left(\begin{array}{c}
2 r \\
i
\end{array}\right) b_{n, k+2 r-i}(t) f(t) \mathrm{d} t \\
& \leq \frac{C n^{r}}{n+1} \sum_{i=0}^{2 r}\left(\begin{array}{c}
2 r \\
i
\end{array}\right) \sum_{k=1}^{\infty} b_{n, k+r}(x) \int_{0}^{\infty} b_{n, k+2 r-i}(t) f(t) \mathrm{d} t .
\end{aligned}
$$

Therefore, by Jensen's inequality [20] and the inequality (2), we obtain

$$
\begin{aligned}
& \left\|\varphi^{2 r}(x) B_{n}^{(2 r)}(f)\right\|_{\Phi} \\
& \quad \leq 2 \inf _{\lambda>0}\left\{\lambda: \int_{0}^{\infty} \Phi\left(\frac{1}{n+1} \sum_{i=0}^{2 r}\left(\begin{array}{c}
2 r \\
i
\end{array}\right) \sum_{k=1}^{\infty} b_{n, k+r}(x) \int_{0}^{\infty} b_{n, k+2 r-i}(t) \frac{C n^{r}|f(t)|}{\lambda} \mathrm{d} t\right) \mathrm{d} x \leq 1\right\} \\
& \quad \leq 2 \inf _{\lambda>0}\left\{\lambda: \int_{0}^{\infty} \frac{1}{2^{2 r}} \sum_{i=0}^{2 r}\left(\begin{array}{c}
2 r \\
i
\end{array}\right) \Phi\left(\frac{1}{n+1} \sum_{k=1}^{\infty} b_{n, k+r}(x) \int_{0}^{\infty} b_{n, k+2 r-i}(t) \frac{C n^{r}|f(t)|}{\lambda} \mathrm{d} t\right) \mathrm{d} x \leq 1\right\} \\
& \quad \leq 2 \inf _{\lambda>0}\left\{\lambda: \int_{0}^{\infty} \frac{1}{2^{2 r}} \sum_{i=0}^{2 r}\left(\begin{array}{c}
2 r \\
i
\end{array}\right) \Phi\left(\frac{1}{n+1} \sum_{k=1}^{\infty} b_{n, k}(x) \int_{0}^{\infty} b_{n, k+r-i}(t) \frac{C n^{r}|f(t)|}{\lambda} \mathrm{d} t\right) \mathrm{d} x \leq 1\right\} \\
& \quad \leq 2 \inf _{\lambda>0}\left\{\lambda: \int_{0}^{\infty} \frac{1}{2^{2 r}} \sum_{i=0}^{2 r}\left(\begin{array}{c}
2 r \\
i
\end{array}\right) \frac{1}{n+1} \sum_{k=1}^{\infty} b_{n, k}(x) \int_{0}^{\infty} b_{n, k+r-i}(t) \Phi\left(\frac{C n^{r}|f(t)|}{\lambda}\right) \mathrm{d} t \mathrm{~d} x \leq 1\right\} \\
& \leq C n^{r}\|f\|_{\Phi,}
\end{aligned}
$$

where $b_{n, k+r-i}(t)=0$ for $n+r-i \leq 0$. Combining this with Equation (5) leads to

$$
\left\|\varphi^{2 r}(x) L_{n, r}^{(2 r)}(f)\right\|_{\Phi} \leq \sum_{i=0}^{2 r-1}\left|c_{i}(n)\right|\left\|\varphi^{2 r}(x) B_{n_{i}}^{(2 r)}(f)\right\|_{\Phi} \leq \sum_{i=0}^{2 r-1}\left|c_{i}(n)\right| C n_{i}^{r}\|f\|_{\Phi} \leq C n^{r}\|f\|_{\Phi} .
$$

Lemma 5 is thus proved.

Lemma 6. Let $f \in L_{\Phi}^{*}[0, \infty)$ and $n \geq 2 r$. Then

$$
\left\|\varphi^{2 r} L_{n, r}^{(2 r)}(f)\right\|_{\Phi} \leq C\left\|\varphi^{2 r} f^{(2 r)}\right\|_{\Phi}
$$

Proof. Integrating by parts $2 r$ times in Equation (10) gives

$$
\begin{aligned}
B_{n}^{(2 r)}(f, x) & =\frac{1}{n+1} \prod_{j=1}^{2 r}(n+j) \sum_{k=1}^{\infty} b_{n+2 r, k}(x) \int_{0}^{\infty} \sum_{i=0}^{2 r}\left(\begin{array}{c}
2 r \\
i
\end{array}\right)(-1)^{i} b_{n, k+2 r-i}(t) f(t) \mathrm{d} t \\
& =\frac{(n+1)(n+2) \cdots(n+2 r)}{n(n-1) \cdots(n-2 r+1)} \frac{1}{n+1} \sum_{k=1}^{\infty} b_{n+2 r, k}(x) \int_{0}^{\infty} b_{n-2 r, k+2 r}^{(2 r)}(t) f(t) \mathrm{d} t \\
& =\frac{(n+2 r) !(n-2 r) !}{(n !)^{2}} \frac{1}{n+1} \sum_{k=1}^{\infty} b_{n+2 r, k}(x) \int_{0}^{\infty} b_{n-2 r, k+2 r}(t) f^{(2 r)}(t) \mathrm{d} t .
\end{aligned}
$$

Accordingly,

$$
\begin{aligned}
\varphi^{2 r}(x) B_{n}^{(2 r)}(f, x) \\
\quad=\frac{(n+2 r) !(n-2 r) !}{(n !)^{2}} \frac{1}{n+1} \sum_{k=1}^{\infty} b_{n+2 r, k}(x) \varphi^{2 r}(x) \int_{0}^{\infty} b_{n-2 r, k+2 r}(t) \varphi^{-2 r}(t) \varphi^{2 r}(t) f^{(2 r)}(t) \mathrm{d} t \\
\quad \leq \frac{C}{n+1} \sum_{k=1}^{\infty} b_{n-2 r+2, k+r}(x) \int_{0}^{\infty} b_{n, k+r}(t) \varphi^{2 r}(t) f^{(2 r)}(t) \mathrm{d} t .
\end{aligned}
$$


Employing Inequality (2) and Jensen's inequality [20] reveals

$$
\begin{aligned}
\| \varphi^{2 r}(x) & B_{n}^{(2 r)}(f) \|_{\Phi} \\
& \leq 2 \inf _{\lambda>0}\left\{\lambda: \int_{0}^{\infty} \Phi\left(\frac{C}{n+1} \sum_{k=1}^{\infty} b_{n-2 r+2, k+r}(x) \int_{0}^{\infty} b_{n, k+r}(t) \frac{\varphi^{2 r}(t)\left|f^{(2 r)}(t)\right|}{\lambda} \mathrm{d} t\right) \mathrm{d} x \leq 1\right\} \\
& \leq 2 \inf _{\lambda>0}\left\{\lambda: \int_{0}^{\infty} \Phi\left(\frac{C}{n+1} \sum_{k=1}^{\infty} b_{n-2 r+2, k}(x) \int_{0}^{\infty} b_{n, k}(t) \frac{\varphi^{2 r}(t)\left|f^{(2 r)}(t)\right|}{\lambda} \mathrm{d} t\right) \mathrm{d} x \leq 1\right\} \\
& \leq 2 \inf _{\lambda>0}\left\{\lambda: \int_{0}^{\infty} \frac{\sum_{k=1}^{\infty} b_{n-2 r+2, k}(x)}{n-2 r+3} \int_{0}^{\infty} b_{n, k}(t) \Phi\left(\frac{\frac{C(n-2 r+3)}{n+1} \varphi^{2 r}(t)\left|f^{(2 r)}(t)\right|}{\lambda}\right) \mathrm{d} t \mathrm{~d} x \leq 1\right\} \\
& \leq 2 \inf _{\lambda>0}\left\{\lambda: \frac{n+1}{n-2 r+3} \int_{0}^{\infty} \Phi\left(\frac{\frac{C(n-2 r+3)}{n+1} \varphi^{2 r}(t)\left|f^{(2 r)}(t)\right|}{\lambda}\right) \mathrm{d} t \leq 1\right\} \\
& \leq C\left\|\varphi^{2 r} f^{(2 r)}\right\|_{\Phi} .
\end{aligned}
$$

Applying the above inequality and Inequality (5) results in

$$
\begin{aligned}
\left\|\varphi^{2 r} L_{n, r}^{(2 r)}(f)\right\|_{\Phi} & =\left\|\sum_{i=0}^{2 r-1} c_{i}(n) \varphi^{2 r} B_{n_{i}}^{(2 r)}(f)\right\|_{\Phi} \leq \sum_{i=0}^{2 r-1}\left|c_{i}(n)\right|\left\|\varphi^{2 r} B_{n_{i}}^{(2 r)}(f)\right\|_{\Phi} \\
\leq C & \sum_{i=0}^{2 r-1}\left|c_{i}(n)\right|\left\|\varphi^{2 r} f^{(2 r)}\right\|_{\Phi} \leq C\left\|\varphi^{2 r} f^{(2 r)}\right\|_{\Phi} .
\end{aligned}
$$

The lemma is proved.

Proof of Theorem 7. From Lemmas 5 and 6 and [21] (Theorem 2.2), we obtain

$$
K_{2 r, \varphi}\left(f, \frac{1}{n^{r / 2}}\right)_{\Phi} \leq \frac{C}{n^{r}} \sum_{k=1}^{n} k^{r-1}\left\|L_{n, r}(f)-f\right\|_{\Phi}
$$

Utilizing Inequality (4) concludes the inverse theorem.

Proof of Theorem 8. Using the so-called order function $\psi(t)=t^{\alpha}|\ln t|^{\beta} e^{|\ln t|^{\gamma}}$ for $0<\alpha<1, \beta \in \mathbb{R}$, and $\gamma<1$ and combining Theorems 7 and 8 conclude the equivalent theorem.

Author Contributions: The authors contributed equally to this work. All authors read and approved the final manuscript.

Funding: This work was partially supported by NSFC (Grant No. 11461052) and IMNSFC (Grant No. 2016MS0118).

Acknowledgments: The authors are thankful to the anonymous referees for their careful corrections to and valuable comments on the original version of this paper.

Conflicts of Interest: The authors declare no conflict of interest.

\section{References}

1. Bardaro, C.; Mantellini, I. On convergence properties for a class of Kantorovich discrete operators. Numer. Funct. Anal. Optim. 2012, 33, 374-396. [CrossRef]

2. Costarelli, D.; Vinti, G. Convergence for a family of neural network operators in Orlicz spaces. Math. Nachr. 2017, 290, 226-235. [CrossRef]

3. Costarelli, D.; Vinti, G. Convergence results for a family of Kantorovich max-product neural network operators in a multivariate setting. Math. Slovaca 2017, 67, 1469-1480. [CrossRef] 
4. Qi, F.; Lim, D.; Guo, B.-N. Explicit formulas and identities for the Bell polynomials and a sequence of polynomials applied to differential equations. Rev. R. Acad. Cienc. Exactas Fís. Nat. Ser. A Math. RACSAM 2018. [CrossRef]

5. Qi, F.; Niu, D.-W.; Guo, B.-N. Some identities for a sequence of unnamed polynomials connected with the Bell polynomials. Rev. R. Acad. Cienc. Exactas Fís. Nat. Ser. A Math. RACSAM 2018, 112. [CrossRef]

6. Yao, Y.; Qin, X.; Yao, J.-C. Projection methods for firmly type nonexpansive operators. J. Nonlinear Convex Anal. 2018, 19, 407-415.

7. Yao, Y.; Yao, J.-C.; Liou, Y.-C.; Postolache, M. Iterative algorithms for split common fixed points of demicontractive operators without priori knowledge of operator norms. Carpathian J. Math. 2018, 34, 459-466.

8. Ueki, S.-I. On the Li-Stević integral type operators from weighted Bergman spaces into $\beta$-Zygmund spaces. Integral Equ. Oper. Theory 2012, 74, 137-150. [CrossRef]

9. Li, H.; Ma, T. Products of composition operators and integral-type operators from Zygmund-type spaces to $Q_{K}$ spaces. Math. Notes 2016, 99, 261-271. [CrossRef]

10. Liang, Y.-X. Integral-type operators from $F(p, q, s)$ space to $\alpha$-Bloch-Orlicz and $\beta$-Zygmund-Orlicz spaces. Complex Anal. Oper. Theory 2018, 12, 169-194. [CrossRef]

11. Gupta, V.; Yadav, R. Approximation by complex summation-integral type operator in compact disks. Math. Slovaca 2013, 63, 1025-1036. [CrossRef]

12. Vural, İ.; Altın, B.; Yüksel, İ. Schurer generalization of $q$-hybrid summation integral type operators. In Computational Analysis; Springer Proceedings in Mathematics \& Statistics; Springer: Cham, Switzerland, 2016; pp. 179-194. [CrossRef]

13. Govil, N.K.; Gupta, V. Simultaneous Approximation for Stancu-Type Generalization of Certain Summation-Integral-Type Operators. In Analytic Number Theory, Approximation Theory, and Special Functions; Springer: New York, NY, USA, 2014; pp. 531-548.

14. Srivastava, H.M.; Gupta, V. A certain family of summation-integral type operators. Math. Comput. Model. 2003, 37, 1307-1315. [CrossRef]

15. Gupta, V.; Mohapatra, R.N.; Finta, Z. A certain family of mixed summation-integral type operators. Math. Comput. Model. 2005, 42, 181-191. [CrossRef]

16. Han, L.X.; Wu, G. Approximation by modified summation integral type operators in Orlicz spaces. Math. Appl. 2017, 30, 613-622.

17. Rao, M.M.; Ren, Z.D. Theory of Orlicz Space; Monographs and Textbooks in Pure and Applied Mathematics, 146; Marcel Dekker, Inc.: New York, NY, USA, 1991.

18. Han, L.X.; Wu, G. Strong converse inequality of Jacobi weighted simultaneous approximation for gamma operators in Orlicz spaces $L_{\Phi}^{*}(0, \infty)$. Appl. Math. J. Chin. Univ. Ser. A 2016, 31, 366-378. (In Chinese)

19. Ditzian, Z.; Totik, V. Moduli of Smoothness; Springer Series in Computational Mathematics, 9; Springer: New York, NY, USA, 1987, doi:10.1007/978-1-4612-4778-4.

20. Costarelli, D.; Spigler, R. How sharp is the Jensen inequality? J. Inequal. Appl. 2015, 2015, 69. [CrossRef]

21. van Wickeren, E. Weak-type inequalities for Kantorovitch polynomials and related operators. Nederl. Akad. Wetensch. Indag. Math. 1987, 90, 111-120. [CrossRef]

(C) 2018 by the authors. Licensee MDPI, Basel, Switzerland. This article is an open access article distributed under the terms and conditions of the Creative Commons Attribution (CC BY) license (http://creativecommons.org/licenses/by/4.0/). 